\title{
Sense of Agency in Multi-Step Actions
}

\author{
Patricia Garrido-Vásquez ${ }^{1,2}$ and Tanja Rock ${ }^{2}$ \\ ' Department of Psychology, University of Concepción, Concepción, Chile \\ ${ }^{2}$ Department of Experimental Psychology and Cognitive Science, Justus Liebig University Giessen, Giessen, Germany
}

\section{KEYWORDS}

sense of agency

implicit

explicit

sensory attenuation

multi-step actions

ABSTRACT

In our daily lives, we frequently execute actions that require several steps to bring about the outcome. However, investigations on how the sense of agency-the sense of controlling our actions and their outcomes-evolves in multi-step actions are still lacking. The purpose of the present research is to fill this gap. In the present study, the participants executed one-step, two-step, and three-step actions in which one, two, or three keys had to be pressed consecutively to generate a tone. We used sensory attenuation as an implicit measure of the sense of agency. Sensory attenuation means that self-produced sensory effects are perceived as less intense than externally generated effects. In the present experiment, sensory attenuation was measured in a psychophysical paradigm and increased in multi-step actions compared to the one-step action. We also asked the participants to explicitly rate the amount to which they felt that they had generated the tone. Ratings were highest in the one-step condition and dropped for multi-step actions, thus showing the opposite pattern of the sensory attenuation data. We assume that enhanced sensory attenuation in multi-step actions could be due to increased effort or more accurate sensorimotor predictions of action effects. The decrease in explicit ratings for multi-step actions might be attributed to reduced perception of causality.

\section{INTRODUCTION}

Many actions in our daily lives require several steps before the expected outcome occurs, for example writing this paper, shopping at the grocery store, or making coffee. The sense of agency (SoA) refers to the sense of controlling our actions and their effects (Haggard, 2017). However, it is to date unclear how the SoA evolves in the context of multi-step actions.

There are different approaches to measure the SoA. The most direct way is to ask a person whether they caused a certain sensory effect or not, or to use graded evaluations of how strongly a person feels that they have caused a certain effect (e.g., Schwarz et al., 2018; Timm et al., 2016). These techniques belong to the group of explicit agency measures. Although this is a very practical way to measure the SoA, explicit measures may be subject to biases (Wegner \& Wheatley, 1999).
Implicit approaches to agency measure perceptual or neutral changes that may be related to the SoA. One of these is sensory attenuation, which means that sensory effects following one's own actions are perceived as less intense than externally generated sensory effects of the same intensity, or that processing self-generated effects is attenuated at the neural level (Beck et al., 2017; Blakemore et al., 1999; Blakemore et al., 1998; Desantis et al., 2012; Gentsch et al., 2015). It can be measured, for example, by comparing the subjective intensity of internally and externally generated stimuli (Weiss et al., 2011). Another very common approach to measure SoA implicitly is intentional binding: the subjective temporal compression of the action-effect interval for

Corresponding author: Patricia Garrido-Vásquez Schmidt, Department of Psychology, Faculty of Social Sciences, University of Concepción, Barrio Universitario, Concepción, Chile. E-mail: pgarridovas@gmail.com 
voluntary actions (Haggard et al., 2002). This phenomenon has also been termed temporal binding (Dewey \& Knoblich, 2014; Majchrowicz \& Wierzchoń, 2018) or causal binding (Buehner \& Humphreys, 2009; Rohde et al., 2014).

Both implicit and explicit techniques are supposed to measure the SoA. However, some studies that have combined different SoA measures have failed to reveal significant correlations between them (Dewey \& Knoblich, 2014; Saito et al., 2015). Thus, the different methods may capture distinct aspects that all contribute in some way to the agency experience, and several methods should be combined to obtain a more comprehensive picture of the SoA.

Research on the SoA has employed different kinds of actions and action effects. A common approach is to have participants press a key, which leads to some sensory effect (e.g., Barlas \& Obhi, 2014; Haering \& Kiesel, 2014; Haggard et al., 2002; Wang et al., 2017). Some authors have also used more complex actions that trigger an effect, such as mouse movements (Damen et al., 2014), joystick movements (Ebert \& Wegner, 2010; Franck et al., 2001), or line drawings (Asai, 2015). Furthermore, apart from this common one action-one effect sequence, studies have shown intentional binding in (a) effect-actioneffect triads (Yabe et al., 2017), (b) for a second tone that followed the classical keypress-tone sequence in the form of an additional action effect (Ruess et al., 2018), and (c) in longer alternating sequences of voluntary actions and their ensuing effects (Imaizumi et al., 2019). However, none of this research has addressed the question of how the number of action steps influences the SoA for that action.

One could assume that multi-step actions may be perceived as more effortful than one-step actions. Thus, research on how effort during an action influences the SoA may provide some relevant background for the present study. Effort has been manipulated in different ways: (a) increasing physical effort, that is, that more bodily strength had to be used during certain actions (Demanet et al., 2013; Howard et al., 2016; Minohara et al., 2016), (b) increasing mental effort in the context of a dual-task paradigm (Howard et al., 2016), and (c) increasing motor difficulty by using the non-dominant hand (Damen et al., 2014). Increased effort has been associated with increased SoA (Damen et al., 2014; Demanet et al., 2013; Minohara et al., 2016), although one study reported the opposite (Howard et al., 2016). These studies have either employed intentional binding as an implicit approach to SoA (Demanet et al., 2013; Howard et al., 2016) or used explicit agency measures (Damen et al., 2014; Minohara et al., 2016), but not a combination of the two. None of these studies measured sensory attenuation.

\section{The Present Research}

In the present study, we investigated the SoA for multi-step actions. We were interested in how the number of action steps may influence both implicit and explicit measures of the SoA.

As an implicit approach to the SoA, we measured sensory attenuation using a psychophysical approach. The paradigm has been established in previous studies (Sato, 2008; Weiss et al., 2011): Participants press a key, which generates a tone of constant intensity, followed by a comparison tone of the same frequency and duration, which may or may not differ in intensity from the self-generated tone. Participants judge which of the two tones sounded louder to them. The responses are used to calculate the point of subjective equality (PSE), which is the intensity of the comparison tone at which the probability of judging the comparison tone as louder than the self-generated tone is exactly $50 \%$. We also measured the SoA explicitly by including some trials in which participants rated the extent to which they felt that they had generated the tone. These explicit rating trials were included in the learning phase, during which the participants acquired the relationship between actions and their subsequent auditory effects. The inclusion of a learning phase was in line with the procedure of two previous studies on which the present research was based (Sato, 2008; Weiss et al., 2011) and follows the idea that these rather artificial action-effect pairingsas opposed to body-related phenomena such as tickling oneself-have to be learned (Dogge et al., 2019).

We varied the number of action steps between one and three, which means that one, two, or three keys had to be pressed consecutively in order to trigger the self-generated tone. Based on previous studies that measured how effort influences the SoA, we reasoned that more action steps would require higher amounts of effort, simply because more steps need to be taken before an action effect occurs. Therefore, we predicted that both explicit and implicit indicators of SoA would increase across the three action levels, that is, that the subjective ratings of having caused the tone would rise and that the PSEs would decrease with more action steps.

\section{METHODS}

\section{Participants}

Thirty-nine students of Justus Liebig University Giessen participated in the present experiment. One participant was excluded due to a technical failure during the measurement. Two more participants were withdrawn because they showed unrealistic PSE values (63 dB and $22 \mathrm{~dB}$, respectively, while the self-generated tone had an intensity of $74 \mathrm{~dB}$ ), which indicates that they had not understood the task. The remaining 36 participants had a mean age of 23.4 years $(S D=3.1$; range: $19-31$ years), 24 were female. They either participated for course credit or received financial compensation of $€ 8$ for their participation. The study was approved by the ethics committee at the Department of Psychology and Sports Science at Justus Liebig University Giessen. All participants provided written informed consent prior to the experiment.

\section{Stimulus Material}

The equipment consisted of a standard computer, a keyboard, a mouse, and headphones (Sennheiser HD 202). The arrow keys of the keyboard were used to execute the different actions as follows: one-step action: left arrow key; two-step action: left arrow key and down arrow key; and three-step action: left arrow key, down arrow key, and right arrow key. Pure tones with three different frequencies $(500,1000$, and $1500 \mathrm{~Hz}$ ), seven different intensity levels $(71,72,73,74,75,76$, and $77 \mathrm{~dB})$, and 100 


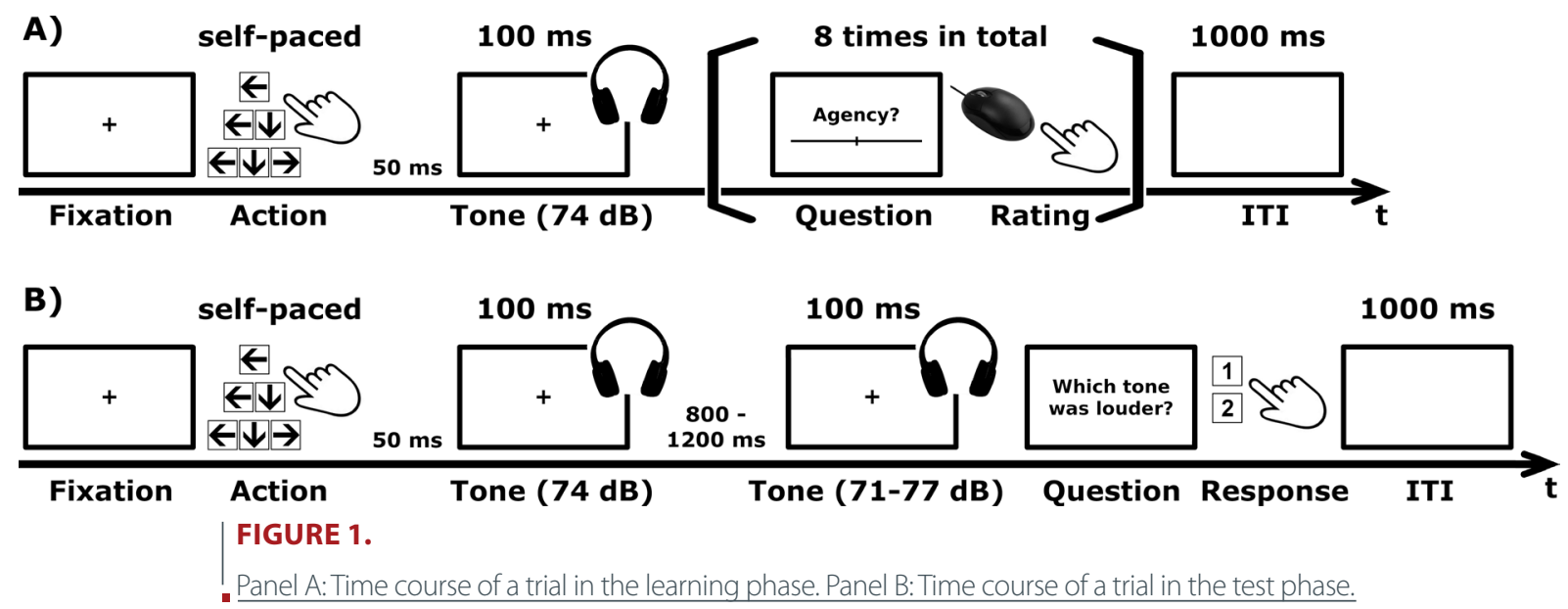

ms duration served as stimuli. Presentation software (Neurobehavioral Systems, San Francisco, USA) was used to run the experiment.

\section{Procedure}

The experiment was presented to the participants as a study investigating tone perception. It consisted of three blocks: a one-step action block, a two-step action block, and a three-step action block. Each block was subdivided into a learning phase, comprising 100 trials, and a test phase, comprising 175 trials, resulting in a total of 825 experimental trials. Block order was assigned randomly to each participant. Furthermore, each tone frequency $(500,1000$, and $1500 \mathrm{~Hz})$ was randomly assigned to one of the blocks for each participant.

Each learning phase started with the instruction that the participant would now acquire the relationship between an action and an event. The participant was asked to freely choose a point in time and execute the corresponding action for the current block, using only the right index finger. Participants were also informed that they would be asked on a regular basis as to how much they felt that they had produced the tone. The instructions for the two- and three-step actions emphasized that the action keys had to be pressed one after another in a speedy manner. If a participant took more than $700 \mathrm{~ms}$ between two keypresses, the program asked the participant to press the keys faster, and the trial was repeated. Actions were followed by a tone of constant intensity (74 dB). The inter-trial interval (ITI) was $1 \mathrm{~s}$.

After 20, 30, 40, 50, 60, 70, 80, and 90 trials (eight times in total during the learning phase), an explicit agency judgment was acquired. In these cases, the tone was followed by a screen asking participants how much they felt that they had produced the tone. Ratings were provided on a continuous scale with the verbal label "not at all" on the left and "very much" on the right, using the left mouse button to move the cursor, which was initially placed at the center of the scale. Figure 1, Panel A shows the procedure of a trial in the learning phase.

The test phase had a similar procedure. However, the self-generated $74 \mathrm{~dB}$ tone was followed by a second tone after a variable delay. The second tone had the same frequency and duration as the self-generated one, but varied randomly in intensity $(71-77 \mathrm{~dB})$. The delay between the two tones varied randomly between 800 and 1200 ms. After the second tone, the participants decided which one was louder using the " 1 " or " 2 " key on the left side of the computer keyboard. The ITI lasted 1 s. Figure 1, Panel B shows the procedure of a trial in the test phase. In total, the experiment lasted about an hour. After the experiment, the participants were thanked for their participation and debriefed.

\section{Data Analysis}

The only independent variable in this experiment is the number of action steps: one, two, or three. To measure sensory attenuation, we calculated the PSEs in decibels as our dependent variable. For the explicit measurement, we used the agency ratings translated into percentages, ranging from 0 to $100 \%$.

The PSE values were calculated as follows: for each intensity level of the comparison tone, the proportion of "second tone louder" responses was determined separately for each of the three action conditions. A logistic function was then fit to the data using a maximum-likelihood procedure, as implemented in the Palamedes toolbox (www.palamedestoolbox.org; Prins \& Kingdom, 2009) running on Matlab (The MathWorks, Natick, USA). To check whether potential PSE differences between conditions could be attributed to differences in perceptual sensitivity, we also calculated the just noticeable difference (JND) for the three actions by subtracting the intensity of the comparison tone judged as louder in $25 \%$ of the trials from the intensity of the comparison tone judged as louder in $75 \%$ of the trials, divided by 2 . Raw data are available from the Open Science Framework, doi: 10.17605/OSF. IO/WKVG4.

\section{RESULTS}

Table 1 and Figure 2 show the sensory attenuation results. To test our hypothesis of stronger sensory attenuation in multi-step actions, we calculated a repeated-measures analysis of variance (ANOVA) on the PSEs. The result (Greenhouse-Geisser corrected) was significant, $F(1.645,57.591)=3.385, p=.05, \eta_{\mathrm{p}}^{2}=.088$. The difference contrast (reverse Helmert contrast) revealed that the PSE in the three-step action was significantly lower than in both the one-step and two-step actions, 


\begin{tabular}{lccc} 
& \multicolumn{1}{l}{$\begin{array}{l}\text { TABLE 1. } \\
\text { Descriptive Statistics }\end{array}$} & 3-step action \\
\hline & 1-step action & 2-step action & 73.51 (0.82) \\
\hline $\begin{array}{l}\text { Point of subjective equality (dB) } \\
\text { Mean }(S D)\end{array}$ & $73.81(0.52)$ & $73.77(0.63)$ & $0.90(0.37)$ \\
Just noticeable difference (dB) & $0.91(0.39)$ & $0.94(0.34)$ & $79.85(26.01)$ \\
$\quad$ Mean $(S D)$ & $83.07(28.10)$ & $79.27(24.81)$ & 94.13 \\
Explicit rating & 97.34 & 92.13 & \\
$\quad \begin{array}{l}\text { Mean }(S D) \\
\text { Median }\end{array}$ & & & \\
\hline
\end{tabular}

$t=2.586, p=.012$ (contrast: three-step vs. one-step and two-step conditions), while the PSEs in the one- and two-step actions did not differ significantly ( $p=.775$; contrast: one-step vs. two-step condition). Thus, sensory attenuation increased with more action steps, but this effect was driven by the three-step action. For detailed information on the proportion of "second tone louder" responses for each comparison tone intensity, separated by action condition, see Table 2 .

An additional repeated-measures ANOVA on JND values revealed no significant difference between the three conditions $(p>$ .776; see Table 1 for the descriptive values).

Explicit agency judgments were higher in the one-step condition than in the other two (see Table 1), and the data were left-skewed (i.e., most ratings were provided on the right side of the scale). Due to strong deviations from normality, we used the non-parametric Friedman test, which revealed a significant result, $\chi^{2}=6.687, p=$ .035. To further analyze this effect, we conducted a Wilcoxon signedrank tests to compare the three conditions. Since the descriptive data pointed in the opposite direction to what we had hypothesized, we used the two-sided test (Pagano, 2009). There was a significant difference between the one- and two-step actions, $Z=419.5, p=.013$. Other comparisons were not significant ( $p$ s > .34).

While timing in the one-step condition was constant (i.e., one button press followed by a tone $50 \mathrm{~ms}$ later), pressing two or three keys in a row could introduce some additional temporal variation due to different delays between keypresses. Therefore, we also analyzed the relationship between this temporal variation and the three outcomes: PSE, explicit rating, and JND. For the two-step action, we calculated the $S D$ of the delays between the first and the second keypress $(M=37.89 \mathrm{~ms}, S D=13.63 \mathrm{~ms}$, range: $14.29-65.54 \mathrm{~ms})$ for each participant. For the three-step action, we calculated the $S D$ s between the first and second $(M=31.63 \mathrm{~ms}, S D=14.03 \mathrm{~ms}$, range: $14.59-73.39 \mathrm{~ms}$ ), and between the second and the third keypress ( $M$ $=32.28 \mathrm{~ms}, S D=14.45 \mathrm{~ms}$, range: $13.26-66.31 \mathrm{~ms}$ ), and correlated these values with our three outcome variables. This procedure was derived from Weiss et al. (2011). None of these correlations were significant $(p s>.43)$.

To sum up, our findings indicate that sensory attenuation-as an implicit indicator of the SoA-increases in multi-step actions while explicit agency judgments are reduced.

\section{DISCUSSION}

The present study investigated how the SoA evolves in multi-step actions. We used actions requiring one, two, or three steps (keypresses), and showed that sensory attenuation for self-generated tones was stronger in multi-step actions than in the one-step action, while explicit agency ratings decreased in multi-step actions as compared to the one-step action. The JND analysis showed that the differences in sensory attenuation could not be attributed to differences in perceptual sensitivity between the three action conditions.

Our findings with respect to sensory attenuation, which is considered an implicit measure of SoA, confirmed our initial predictions based on research showing a stronger SoA for more effortful actions (Damen et al., 2014; Demanet et al., 2013; Minohara et al., 2016). Thus, one explanation for the present results could be that multi-step actions are experienced as more effortful than the one-step action. However, since we did not measure subjective effort, this explanation will need to be addressed in future research.

An alternative explanation for our results could be that sensorimotor prediction was more accurate in multi-step actions than in the one-step action. Previous research has shown that as stimuli become more predictable, sensory attenuation increases, even when the stimuli are not self-generated (Kaiser \& Schütz-Bosbach, 2018). In

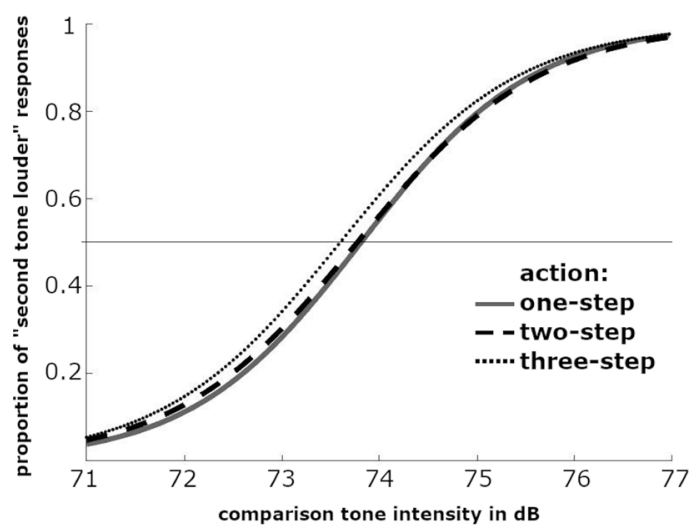

FIGURE 2.

Psychometric curves averaged across all participants for each of the three conditions. The horizontal line indicates the PSE (probability of "second tone louder" responses $=50 \%$ ). 


\begin{tabular}{lccc}
\hline $\begin{array}{l}\text { TABLE 2. } \\
\text { Proportion of "Second Tone Louder" Responses for Each Com- } \\
\text { parison Tone Intensity, Separated by Action Condition }\end{array}$ \\
\hline $\begin{array}{c}\text { Comparison tone } \\
\text { intensity }\end{array}$ & 1-step action & 2-step action & 3-step action \\
\hline $71 \mathrm{~dB}$ & .07 & .06 & .08 \\
$72 \mathrm{~dB}$ & .10 & .13 & .13 \\
$73 \mathrm{~dB}$ & .24 & .27 & .33 \\
$74 \mathrm{~dB}$ & .55 & .56 & .60 \\
$75 \mathrm{~dB}$ & .83 & .82 & .83 \\
$76 \mathrm{~dB}$ & .93 & .91 & .94 \\
$77 \mathrm{~dB}$ & .97 & .96 & .98 \\
\hline
\end{tabular}

fact, latter study which measured N1 reduction in the event-related potential (ERP) as an indicator for sensory attenuation reported that N1 reduction for the condition in which a three-step countdown (but no participant action) preceded the effect was greater than when the action effect had been generated by a single button press from the participant. Another ERP study using a similar procedure reported a seemingly contradictory result, with self-generated sounds resulting in stronger $\mathrm{N} 1$ attenuation than fully predictable but externally generated sounds (Klaffehn et al., 2019). A key difference between both studies could be the way in which the externally generated effect was made predictable: In the study by Klaffehn et al. (2019), a filling-up bar was presented on screen, while Kaiser and Schütz-Bosbach (2018) used a visual countdown (3-2-1) to announce the externally generated effect. This rather rhythmic way of announcing an effect may be key to increased sensory attenuation, potentially due to a temporal expectancy effect (Jones et al., 2017). Future research will have to further explore the possibility of more accurate sensorimotor predictions in the context of multi-step actions and how they come about.

Contrary to our predictions, explicit agency judgments were reduced in multi-step actions. This finding is at odds with the literature on the relationship between effort and the SoA, which reported increased judgments of agency for more effortful actions (Damen et al., 2014; Minohara et al., 2016). The effect cannot be explained by increased temporal variability between keypresses in the two- and three-step actions because the correlation between temporal variability and explicit agency judgments was not significant. We propose that the diminished judgments of agency in multi-step actions could be due to a reduced perception of causality, one of the key mechanisms of the SoA (Desantis et al., 2011). Potentially, if an action consists of several steps, the perceived likelihood that mechanisms beyond the person's actions could play a role in generating the observed action effects increases (Wegner \& Wheatley, 1999) because "more "is happening" between action initiation and the ensuing effect. Therefore, causality should be manipulated in future studies in order to shed more light on this explanation.

Our results also add to the evidence showing that implicit and explicit agency measures capture different aspects of the SoA (Dewey \& Knoblich, 2014; Saito et al., 2015). Results from implicit and explicit agency measurements pointed into opposite directions, in line with some previous findings (Majchrowicz \& Wierzchoń, 2018; Wen et al., 2015). However, due to the temporal separation of explicit (learning phase) and implicit (test phase) measurements in the present study, these results should be interpreted with caution, because temporal proximity between implicit and explicit agency measures may abolish potential dissociations between these two types of measures (Imaizumi \& Tanno, 2019).

Adopting the idea of reduced causality perception with more action steps, we cannot currently discard the possibility that the sensory attenuation effects observed in the present experiment captured something else than an implicit measure of the SoA. If causality perception indeed decreases with more action steps, an implicit measure of SoA should also reflect this. In fact, both sensory attenuation and intentional binding have been questioned regarding their ability to adequately capture the SoA, because they have also been observed outside the context of voluntary actions (Kaiser \& Schütz-Bosbach, 2018; Kirsch et al., 2019; Suzuki et al., 2019). Thus, in line with some arguments outlined above, the present sensory attenuation effects may be a consequence of better temporal predictability of the tone in the three-step action, rather than reflecting a stronger SoA.

One key limitation to the present study is that more action steps also implied longer delays between action initiation and the corresponding action effects. Our results suggest a dissociation between the action-effect delay on the one hand and the action initiation-effect delay on the other. Longer action-effect delays have been associated with reduced SoA (Blakemore et al., 1999; Elijah et al., 2016), while our sensory attenuation data indicate an increase of this implicit SoA measure at longer action initiation-effect delays. More complex designs are needed to isolate the effects of the number of action steps on the one hand from the duration of the action initiation-effect delay on the other.

\section{ACKNOWLEDGEMENTS}

The authors would like to thank two anonymous reviewers for helpful comments on a previous version of the present manuscript.

\section{REFERENCES}

Asai, T. (2015). Feedback control of one's own action: Self-other sensory attribution in motor control. Consciousness and

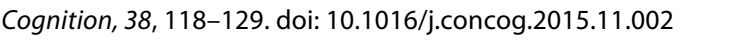

Barlas, Z., \& Obhi, S. S. (2014). Cultural background influences implicit but not explicit sense of agency for the production of musical tones. Consciousness and Cognition, 28, 94-103. doi: 10.1016/j.concog.2014.06.013 1.

Beck, B., Di Costa, S., \& Haggard, P. (2017). Having control over the external world increases the implicit sense of agency. Cognition, 162, 54-60. doi: https://doi.org/10.1016/j.cognition.2017.02.002 1لس

Blakemore, S. J., Frith, C. D., \& Wolpert, D. M. (1999). Spatiotemporal prediction modulates the perception of self-produced stimuli. Journal of Cognitive Neuroscience, 11, 551-559. doi: 10.1162/089892999563607 
Blakemore, S. J., Wolpert, D. M., \& Frith, C. D. (1998). Central cancellation of self-produced tickle sensation. Nature Neuroscience

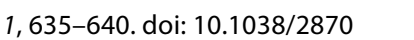

Buehner, M. J., \& Humphreys, G. R. (2009). Causal binding of actions to their effects. Psychological Science, 20, 1221-1228. doi: 10.1111/j.1467-9280.2009.02435.x السلسلس

Damen, T. G., Dijksterhuis, A., \& Baaren, R. B. v. (2014). On the other hand: Nondominant hand use increases sense of agency. Social Psychological and Personality Science, 5, 680-683. doi: 10.1177/1948550614527626 10

Demanet, J., Muhle-Karbe, P. S., Lynn, M. T., Blotenberg, I., \& Brass, M. (2013). Power to the will: How exerting physical effort boosts the sense of agency. Cognition, 129, 574-578. doi:

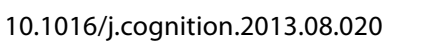

Desantis, A., Roussel, C., \&Waszak, F. (2011). On the influence of causal beliefs on the feeling of agency. Consciousness and Cognition, 20, 1211-1220. doi: 10.1016/j.concog.2011.02.012 المالسلسلس

Desantis, A., Weiss, C., Schütz-Bosbach, S., \& Waszak, F. (2012). Believing and perceiving: Authorship belief modulates sensory attenuation. PLoS One, 7, e37959. doi: 10.1371/journal. pone.0037959 السلسلس

Dewey, J. A., \& Knoblich, G. (2014). Do implicit and explicit measures of the sense of agency measure the same thing? PLOS One, 9, e110118. doi: 10.1371/journal.pone.0110118 السلسلس

Dogge, M., Custers, R., \& Aarts, H. (2019). Moving forward: On the limits of motor-based forward models. Trends in Cognitive Sciences, 23, 743-753. doi: 10.1016/j.tics.2019.06.008 السلسلسا Cogniti

Ebert, J. P., \& Wegner, D. M. (2010). Time warp: Authorship shapes the perceived timing of actions and events. Consciousness and Cognition, 19, 481-489. doi: 10.1016/j.concog.2009.10.002 الس السلس

Elijah, R. B., Le Pelley, M. E., \& Whitford, T. J. (2016). Modifying temporal expectations: Changing cortical responsivity to delayed self-initiated sensations with training. Biological Psychology, 120, 88-95. doi: 10.1016/j.biopsycho.2016.09.001 السلسلس

Franck, N., Farrer, C., Georgieff, N., Marie-Cardine, M., Dalery, J., d'Amato, T., \& Jeannerod, M. (2001). Defective recognition of one's own actions in patients with schizophrenia. American Journal of Psychiatry, 158, 454-459. doi: 10.1176/appi. ajp.158.3.454 السلسلس

Gentsch, A., Weiss, C., Spengler, S., Synofzik, M., \& SchützBosbach, S. (2015). Doing good or bad: How interactions between action and emotion expectations shape the sense of agency. Social Neuroscience, 10, 418-430. doi: 10.1080/17470919.2015.1006374 السلسلس

Haering, C., \& Kiesel, A. (2014). Intentional binding is independent of the validity of the action effect's identity. Acta Psychologica, 152, 109-119. doi: 10.1016/j.actpsy.2014.07.015 الهالسلسلسا

Haggard, P. (2017). Sense of agency in the human brain. Nature Reviews Neuroscience, 18, 196-207. doi: 10.1038/nrn.2017.14 السلسلسلة

Haggard, P., Clark, S., \& Kalogeras, J. (2002). Voluntary action and conscious awareness. Nature Neuroscience, 5, 382-385. doi: 10.1038/nn827 سلس
Howard, E. E., Edwards, S. G., \& Bayliss, A. P. (2016). Physical and mental effort disrupts the implicit sense of agency. Cognition, 157, 114-125. doi: 10.1016/j.cognition.2016.08.018 المالسلسل|

Imaizumi, S., \& Tanno, Y. (2019). Intentional binding coincides with explicit sense of agency. Consciousness and Cognition, 67, 1-15. doi: 10.1016/j.concog.2018.11.005 السلسلس

Imaizumi, S., Tanno, Y., \& Imamizu, H. (2019). Compress global, dilate local: Intentional binding in action-outcome alternations. Consciousness and Cognition, 73, 102768. doi: 10.1016/j. concog.2019.102768 سلسلسلس

Jones, A., Hsu, Y.-F., Granjon, L., \& Waszak, F. (2017). Temporal expectancies driven by self- and externally generated rhythms. Neurolmage, 156, 352-362. doi: 10.1016/j.neuroimage.2017.05.042 السلسلس

Kaiser, J., \& Schütz-Bosbach, S. (2018). Sensory attenuation of self-produced signals does not rely on self-specific motor predictions. European Journal of Neuroscience, 47, 1303-1310. doi: 10.1111/ejn.13931 المالسلس

Kirsch, W., Kunde, W., \& Herbort, O. (2019). Intentional binding is unrelated to action intention. Journal of Experimental Psychology: Human Perception and Performance, 45, 378-385. doi: 10.1037/xhp0000612 المالملكسل

Klaffehn, A. L., Baess, P., Kunde, W., \& Pfister, R. (2019). Sensory attenuation prevails when controlling for temporal predictability of self- and externally generated tones. Neuropsychologia, 132,

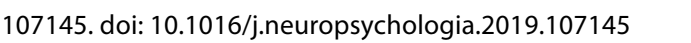

Majchrowicz, B., \& Wierzchoń, M. (2018). Unexpected action outcomes produce enhanced temporal binding but diminished judgement of agency. Consciousness and Cognition, 65, 310-324. doi: 10.1016/j.concog.2018.09.007 الس الس الس

Minohara, R., Wen, W., Hamasaki, S., Maeda, T., Kato, M., Yamakawa, H., ... Asama, H. (2016). Strength of intentional effort enhances the sense of agency. Frontiers in Psychology, 7, 1165. doi: 10.3389/fpsyg.2016.01165 السلسلس

Pagano, R. R. (2009). Understanding statistics in the behavioral sciences (9th ed.). Cengage Learning.

Prins, N., \& Kingdom, F. A. A. (2009). Palamedes: MATLAB routines for analyzing psychophysical data. Retrieved from http://www. palamedestoolbox.org/download.html

Rohde, M., Greiner, L., \& Ernst, M. O. (2014). Asymmetries in visuomotor recalibration of time perception: Does causal binding distort the window of integration? Acta Psychologica, 147, 127-135. doi: 10.1016/j.actpsy.2013.07.011 المالسلسلس

Ruess, M., Thomaschke, R., Haering, C., Wenke, D., \& Kiesel, A. (2018). Intentional binding of two effects. Psychological Research, 82, 1102-1112. doi: 10.1007/s00426-017-0892-4 الس السلسلة

Saito, N., Takahata, K., Murai, T., \& Takahashi, H. (2015). Discrepancy between explicit judgement of agency and implicit feeling of agency: Implications for sense of agency and its disorders. Consciousness and Cognition, 37, 1-7. doi: 10.1016/j.concog.2015.07.011 الس السلسل

Sato, A. (2008). Action observation modulates auditory perception of 
the consequence of others' actions. Consciousness and Cognition, 17, 1219-1227. doi: 10.1016/j.concog.2008.01.003 الس الس الس الس

Schwarz, K. A., Burger, S., Dignath, D., Kunde, W., \& Pfister, R. (2018). Action-effect binding and agency. Consciousness and Cognition, 65, 304-309. doi: 10.1016/j.concog.2018.10.001 سلس Suzuki, K., Lush, P., Seth, A. K., \& Roseboom, W. (2019). Intentional binding without intentional action. Psychological Science, 30 , 842-853. doi: 10.1177/0956797619842191 الس السلس

Timm, J., Schönwiesner, M., Schröger, E., \& SanMiguel, I. (2016). Sensory suppression of brain responses to self-generated sounds is observed with and without the perception of agency. Cortex, 80, 5-20. doi: 10.1016/j.cortex.2016.03.018 الس الس

Wang, Y., Damen, T. G. E., \& Aarts, H. (2017). Uncovering effects of self-control and stimulus-driven action selection on the sense of agency. Consciousness and Cognition, 55, 245-253. doi:

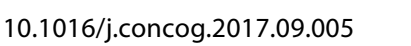

Wegner, D. M., \& Wheatley, T. (1999). Apparent mental causation. Sources of the experience of will. The American Psychologist, 54, 480-492. doi: 10.1037/0003-066X.54.7.480 الس الس الس

Weiss, C., Herwig, A., \& Schütz-Bosbach, S. (2011). The self in action effects: selective attenuation of self-generated sounds. Cognition,

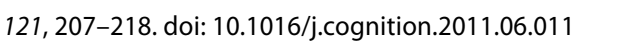

Wen, W., Yamashita, A., \& Asama, H. (2015). The influence of action-outcome delay and arousal on sense of agency and the intentional binding effect. Consciousness and Cognition, 36, 87-95. doi: 10.1016/j.concog.2015.06.004 السلسلس

Yabe, Y., Dave, H., \& Goodale, M. A. (2017). Temporal distortion in the perception of actions and events. Cognition, 158, 1-9. 10.1016/j.cognition.2016.10.009 الس الس

RECEIVED 04.11.2019| ACCEPTED 24.01.2020 